\title{
Toward Proton Computed Tomography
}

\author{
Hartmut F.-W. Sadrozinski, Senior Member, IEEE, Vladimir Bashkirov, Brian Keeney, Leah R. Johnson, \\ Stephen G. Peggs, Gabe Ross, Todd Satogata, Reinhard W. M. Schulte, Abraham Seiden, Kabiz Shanazi, and \\ David C. Williams
}

\begin{abstract}
Proton therapy, long regarded as a superior method of radiation therapy, is now becoming more cost effective and is being used in a number of clinical centers around the world. In light of this development the use of the proton beam itself should be considered for the most accurate method of treatment planning. $\mathrm{X}$-ray computed tomography (XCT), which is widely available, has been used for the treatment planning for proton therapy. The basic interactions of XCT in matter are fundamentally different than those of the protons. Thus, the resulting density map from XCT is only an approximation of the true density map for proton therapy. Progress in proton computed tomography (pCT) is presented in this work. The experimental requirements for pCT are examined, and data analysis and Monte Carlo simulations are used to estimate the feasibility of pCT as an imaging modality.
\end{abstract}

Index Terms-Proton radiation effects, tomography.

\section{INTRODUCTION}

$\mathbf{X}$ -RAY computed tomography (XCT) is the preferred technique for planning the treatment of patients for proton radiation therapy. In XCT the measurement is performed with a continuum of X-rays produced as Bremstrahlung from a relatively standard X-ray tube. The resulting image is a density map corresponding to a nonlinear average of the continuum of $\mathrm{X}$-rays, which may be absorbed or scattered depending on the density and effective atomic number of the body part.

Proton radiation therapy is a precise form of radiation therapy. Avoidance of damage to critical normal tissues and prevention of geographical tumor misses require accurate knowledge of the dose delivered to the patient and verification of the correct patient position with respect to the proton beam. In existing proton treatment centers, dose calculations are performed based on XCT and the patient is positioned with $\mathrm{X}$-ray radiographs [1]. However, the use of XCT images for proton treatment planning ignores fundamental differences in physical interaction processes between photons and protons and is, therefore, potentially inaccurate. Further, X-ray radiographs mainly depict patients' skeletal structures and rarely show the tumor itself. Ideally, one would image the patient directly with

Manuscript received August 11, 2003; revised November 10, 2003. This work was supported by DoE and Calspace.

H. F.-W. Sadrozinski, B. Keeney, L. R. Johnson, G. Ross, A. Seiden, and D. C. Williams are with the Santa Cruz Institute for Particle Physics, University of California, Santa Cruz, CA 95064 USA (e-mail: hartmut@scipp.ucsc.edu; bkeeney@scipp.ucsc.edu; leah@scipp.ucsc.edu; gabe@scipp.ucsc.edu; abs@scipp.ucsc.edu; davidw@scipp.ucsc.edu).

V. Bashkirov, R. W. M. Schulte, and K. Shanazi are with the Department of Radiation Medicine, Loma Linda University Medical Center, Loma Linda, CA 92354 USA (e-mail: vbashkirov@dominion.llumc.edu; rschulte@dominion.llumc.edu; kshanazi@dominion.llumc.edu).

S. G. Peggs and T. Satogata are with the Brookhaven National Laboratory, Upton, NY 11973 USA (e-mail: peggs@bnl.gov; satogata@bnl.gov).

Digital Object Identifier 10.1109/TNS.2003.823044 protons, for example, by measuring their energy loss after traversing the patient [2]. This method has the potential to significantly improve the accuracy of proton radiation therapy treatment planning and the alignment of the target volume with the proton beam. Thus there is ongoing interest in the feasibility of proton beam computed tomography (pCT) and proton transmission radiography (PTR).

Here we will briefly review the basic differences between $\mathrm{X}$-ray and proton imaging and the benefits of protons in cancer. Then we will provide a historical overview of $\mathrm{pCT}$ and discuss the requirements for $\mathrm{pCT}$ measurements. In addition to simple absorption measurements a recent experimental and simulation study shows the usefulness of including the angular information of the outgoing proton.

\section{INTERACTION IN MATteR: X-RAYS VERSUS PROTON}

Diagnostic and therapeutic X-rays, which are in the $10-140 \mathrm{KeV}$ range, interact with the imaged primarily by Compton scattering wit the electrons. Thus the process is statistical in its form, and one measures the number of transmitted photons. After passing through a thickness $l$, the original number of photons $\mathrm{N}_{0}$ is reduced exponentially to $\mathrm{N}(l)$

$$
N(l)=N_{0} e^{-\mu l}
$$

where $\mu$ is the attenuation coefficient. Fig. 1(a) shows the attenuation coefficient [3] as a function of energy in the relevant $\mathrm{X}$-ray range for medical imaging $(10-140 \mathrm{keV})$ for bone, water, muscle, and fat. The energy dependence is large, and with the exception of bone due to the higher atomic number of $\mathrm{Ca}$, the values of $\mu$ are very close to that of water for the different types of soft tissue. Thus, X-ray images of soft tissue are of low contrast, while bone can be imaged very well. Also, due to the exponential decrease of the numbers of photons as they penetrate the patient, the dose will be highest close to the entrance. Protons with energies used in therapeutic applications ( $70 \mathrm{MeV}-250 \mathrm{MeV}$ ) lose energy mainly through inelastic collisions with atomic electrons as described by the Bethe-Bloch equation [4]. The energy loss $\Delta \mathrm{E}$ is the integral over the specific energy loss along the track length $l$

$$
\Delta E=\int \frac{d E}{d l} d l=\int \frac{d E}{d x} d x \approx \sum \rho(l) \frac{d E}{d x} \Delta l
$$

where $\mathrm{dE} / \mathrm{dx}$ is the "stopping power," the energy loss in the track length element $\Delta l$ weighted by the density $\rho, \mathrm{x}=\rho * l$. The stopping power is a useful concept because it exhibits only a weak dependence on the material traversed, i.e., $\mathrm{dE} / \mathrm{dx} \sim \mathrm{Z} / \mathrm{A}$ [5]. Fig. 1(b) shows the proton specific energy loss $\mathrm{dE} / \mathrm{d} l$ for 
the same materials as in Fig. 1(a) [3]. The energy dependence is fairly strong in the interesting energy range, which can be exploited in experiments to determine the particle energy loss through its final energy [6], [7]. However, in medical applications, the difference in the specific energy loss between different materials is small due to the small difference in density: relative to water, bone has $\Delta \rho=0.5 \mathrm{~g} / \mathrm{cm}^{3}$, and the density difference between fat and muscle is about $\Delta \rho=0.1 \mathrm{~g} / \mathrm{cm} \mathrm{cm}^{3}$. Thus proton CT is inherently low contrast. Equation (2) shows that measuring the energy loss essentially measures the density distribution in the traversed material: the basic interaction leaves the proton intact (unless it undergoes nuclear interaction), The properties of individual particles, energy, and exit position and angle can be measured and hopefully the density distribution of the traversed material deduced more accurately from this additional information.

Protons undergo multiple Coulomb scattering (MCS) while traversing the material. The projected scattering angle is energy and material dependent

$$
\theta_{\mathrm{MCS}} \approx \frac{13.6 \mathrm{MeV}}{\beta p} \mathrm{z} \sqrt{l / X_{0}}
$$

where $\mathrm{z}$ is the charge of the projectile ( $\mathrm{z}=1$ for protons) and $\mathrm{X}_{\mathrm{O}}$ is the radiation length [5]. For a $250 \mathrm{MeV}$ proton traversing $20 \mathrm{~cm}$ of water, the multiple scattering angle is about $\Theta_{\mathrm{MCS}} \approx$ $1^{0}$. This leads to a blurring of the image (see Section VI).

\section{AdVANTAGES OF PROTON THERAPY AND TOMOGRAPHY}

The negative slope of the stopping power curves for proton shown in Fig. 1(b) has important implications for the application of protons in therapy. While traversing the medium, the protons slow down, consequently their $\mathrm{dE} / \mathrm{dx}$ increases until they stop. This is shown in Fig. 2, where the particle energy E and the energy loss $\Delta \mathrm{E}$ for $1 \mathrm{~mm} \Delta \mathrm{x}^{\prime} \mathrm{s}$ are plotted as a function of the proton path length in water. Near the stopping point of their range, the protons lose a large amount of energy over a very small distance and then stop. Thus there is a relatively small entrance dose with a similar dose per $\Delta \mathrm{x}$ for most of the range, and a maximum dose deposition in the region of the proton's stopping point. For extended tumors, the energy of the proton beam is modulated to vary the range across the site of the tumor. In contrast, X-rays tend to have a high skin dose, moderate energy deposition that drops off exponentially at depth, and of course, there is dose in the tissue beyond the tumor. Since there is an increase in the number of low-energy proton accelerators dedicated to therapy [8], it is appropriate to understand the strengths and limitations of the techniques that are to be used in clinical practice. Proton CT can in principle directly measure the density distribution needed in a patient for the dose distribution. On the other hand, the use of X-ray CT for treatment planning in proton cancer therapy has the potential for significant errors in dose distributions. This has been shown in [9], where end point range uncertainties introduced by using XCT as compared to PTR measurements were seen to be larger than $15 \mathrm{~mm}$ in certain locations in the head.

Future work has to show how much pCT can be used in daily clinical treatment planning, due to the practical aspects of $\mathrm{pCT}$,
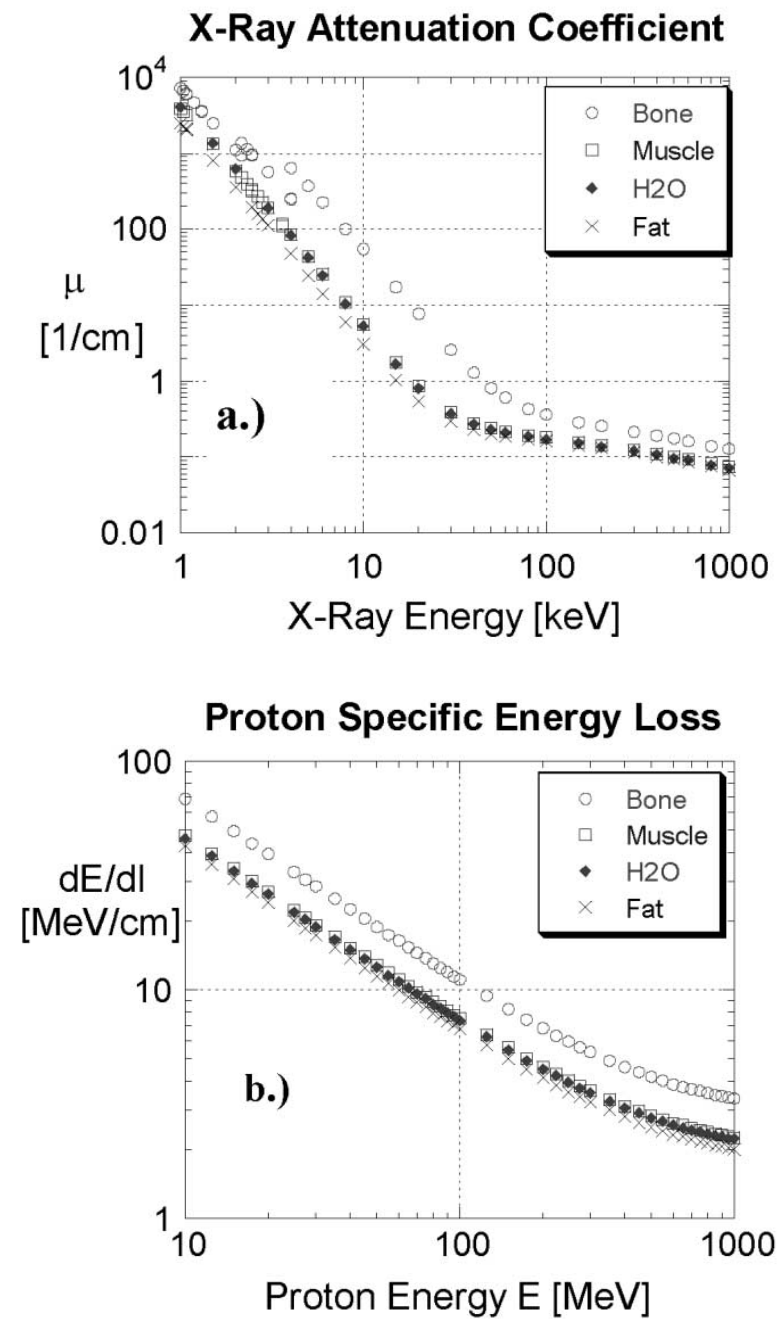

Fig. 1. (a) X-ray attenuation coefficient and (b) proton specific energy loss as a function of energy for bone, muscle, water, and fat [3]. The large contrast for bone in X-rays is due to factor 10 times larger attenuation coefficient with respect to water, while the difference between different tissue and water is relatively small both in X-rays and protons.

but a careful study with pCT versus XCT could be used to develop methods for reducing the errors inherent in XCT.

\section{Milestones in PROTON COMPUTED TomogRaPhy}

\section{A. Initial Development}

The field of hadron therapy started with the observation by Wilson in 1946 [10] that the Bragg peak and well defined range of mono-energetic hadrons could be used for precision treatment of cancer. Wilson's vision of using either existing HEP accelerators or dedicated medical hadron machines for cancer treatment has been pursued ever since [8]. The use of protons for imaging ("Tomography") was advocated by Cormack in 1963 [11], and in 1972, Goitein [12] elucidated the methods for acquiring the appropriate 2-D data and its reconstruction into 3-D images, backing it up by simulations. Cormack and Koehler [13] showed in 1976, that pCT can detect extremely low-contrast features by imaging sugar solutions with a density difference of $\Delta \rho \ll 0.5 \%$ relative to water. The LANL Group of Hanson 


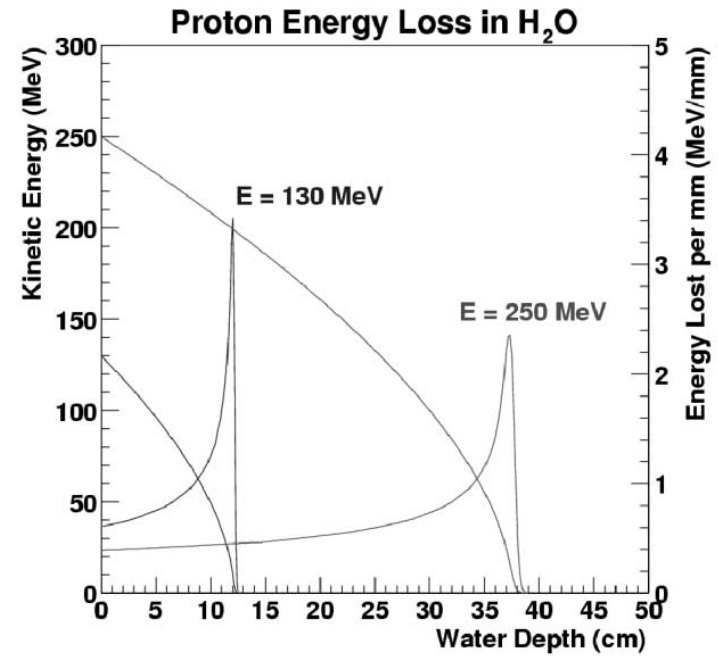

Fig. 2. Proton energy loss in water as a function of penetration depth for two incident proton energies. The left hand scale indicates the energy of the protons and the right hand scale the energy deposited in $1 \mathrm{~mm}$ water. The energy deposit is characterized by an entrance plateau, and the so-called Bragg peak at the end of the range, which is used in proton beam treatment to deliver maximum dose to the tumor. Beyond the well-defined range the intensity exhibits a rapid distal falloff. In Proton CT, the energy loss of protons in the plateau will be used, which will minimize the dose to the patient. (Note the increased width of the Bragg peak with increased energy.)

et al. [14] showed imaging of human tissue using a high precision range telescope in 1982. In their study they pointed out, that $\mathrm{pCT}$ has a potential dose advantage with respect to XCT when imaging tissue, which has inherently low contrast in both methods. Recently, the PSI group of Schneider et al. [15] has been very active in both calibrating CT values, and in developing a working pCT system. The BNL group [16] also pointed out this potential dose advantage of pCT.

\section{B. Current Progress in $p C T$}

There has been an increase in the number of dedicated accelerator facilities with gantry beam delivery systems [8] for clinical procedures. There is interest in academia with fairly recent Ph.D. theses at PSI [17], [18] and the Harvard Cyclotron [19], which investigate the theory and practical aspects of $\mathrm{pCT}$. The availability of high bandwidth detector systems for the detection of the protons, which will reduce the beam time required for the measurement, may provide the pathway for practical clinical evaluation of $\mathrm{pCT}$. Semiconductor detectors are now available, which have count rate capability in excess of $10^{6} \mathrm{cps}$. These detectors can be produce as a 2-D array on 6" wafers with 50-100 micron pitch. Such a detector can localize precisely the proton exiting the body and measure its energy with moderate precision. There is a growing interest in Monte Carlo simulation of pCT. These models take advantage of the correlation between scatter angle and energy, and have potential for developing the entire proton imaging and treatment process, including the optimization of the beam energy, design of the pCT instrumentation, data analysis, image reconstruction and dose calculation and minimization.

\section{Proton-By-Proton Track ReCONSTRUCtion}

With the current level of development of arrays or silicon strip detectors, proton-by-proton data collection should be pos- sible at the accuracy and high rates necessary for pCT. A typical 300 micron Si detector will absorb about 0.3 to $2 \mathrm{MeV}$ from impinging 150 to $50 \mathrm{MeV}$ protons. This measurement would only give the deflection of the proton in the body. In order to measure the angle, it will be necessary to have a stack of two silicon detectors with very good spatial resolutions and this could be backed up by a large absorbing detector to get the total energy of the proton. The position, exit angle and the total energy of the proton is essentially all the information that can be gleaned from the exiting proton. The fixed beam geometry provides the reference coordinate system for the measurement. A prototype proton-by-proton "camera" has been installed on the PSI proton therapy gantry, and early operational performance has been reported, albeit without track reconstruction. For example, Schneider and Pedroni [20] show radiographic projections produced by plotting the average exit range of protons, versus the transverse $x$ and $y$ coordinates measured in the entry plane. They also show "edge enhanced" images taken with the same data, when the rms exit range variation is plotted versus entrance $x$ and $y$-making use of the multiple scattering phenomenon. In a manner similar to the iterative reconstruction programs used in PET and SPECT, the detailed information from the detector, such as the angle of deflection of the proton and the final energy of the proton can be modeled, using the basic physics of the process [6], [7], [28].

\section{Multiple Coulomb Scattering}

In addition to energy, the incident proton beam will spread out through the process of multiple Coulomb scattering (MCS), cf. (3), even if the incoming beam had zero size and zero angular spread. For example, a $200 \mathrm{MeV}$ beam will acquire an rms transverse size of $6.5 \mathrm{~mm}$ by the end of its range in water. The spatial resolution of the radiographic image is defined by the rms size of the beam on exit, if proton-by-proton track reconstruction is not possible, or is not performed. Typically, this is a few millimeters, unacceptably large in comparison with the submillimeter resolution possible with X-ray CT, MRI, or even with PET imaging.

However, the acquisition of proton-by-proton displacements and angles at entry and exit can assist in defining the location of the internal trajectory of each proton, perhaps at the submillimeter level. The imaging problem is reduced to answering the question "what is the most probable internal trajectory" for each proton, and what is the statistical uncertainty around the mean. In the simple case of a water phantom, it is possible to answer these questions analytically, even with a crude exit displacement measurement, and no exit angle measurement [20]. When both particle position and angle are measured at the entrance and exit, the prediction of the most probable trajectory and its uncertainty are sharpened considerably [21]. This is illustrated in Fig. 3, which shows the most likely trajectories and their 1-sigma and 2-sigma envelope for identical entrance parameters, but two exit positions and two exit angles - The width of the 'bananas' is less than a millimeter, and the knowledge of the exit angle locates the internal trajectory to submillimeter accuracy [21]. 

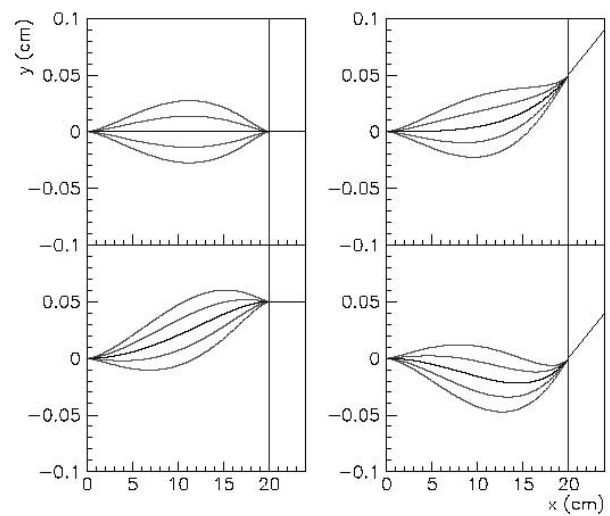

Fig. 3. Most likely trajectories and one and two sigma envelopes for protons with the same entrance position and angle, but different exit position and angle [21]. The measurement of the exit angles allows considerable improvement in the positioning of the "banana" inside the material.

\section{SENSITIVITY}

The consequences of the low contrast in $\mathrm{pCT}$ can be shown in a simple one-dimensional sensitivity study using the energy loss data tabulated by NIST [3]. We approximate tissue with a $20 \mathrm{~cm}$ deep water column of large sideways dimension, and imbed at the midpoint an inclusion layer of thickness $1=1 \mathrm{~cm}$. The density $\rho$ of the layer is varied from 1.0 to $1.1,1.5$, and $2.0 \mathrm{~g} / \mathrm{cm}^{3}$. The effect of the difference in energy loss on $250 \mathrm{MeV}$ protons is shown in Fig. 4. In Fig. 4(a), the average energy loss in $1 \mathrm{~mm}$ water is shown as a function of water depth, showing the entrance plateau and the increased energy loss in the $1 \mathrm{~cm}$ long inclusion depending on the density selected. In Fig. 4(b), the proton energy is plotted as a function of water depth for the four densities of the inclusion, showing only a slight dispersion in energy at the exit at $20 \mathrm{~cm}$ depth. Fig. 4(c) shows the difference in energy as a fraction of the energy, indicating a few $\%$ for the high contrast image and fraction of a $\%$ for a typical low contrast soft tissue. Table I shows the attributes of the exiting protons which could be measured as a function of the density, for different $\mathrm{x}=\rho * \mathrm{l}$. Again, the differences are very small: for a $10 \%$ density increase, the exit energy changes by $0.5 \mathrm{MeV}$, the range by $1 \mathrm{~mm}$, and the TOF in a $20 \mathrm{~cm}$ TOF system by 2 ps. The energy straggling in $20 \mathrm{~cm}$ water is about $1 \mathrm{MeV}$, and the corresponding range straggling about $2 \mathrm{~mm}$, which means that the energy difference in the low contrast case can only be determined statistically (see Section VIII).

\section{REQUIREMENTS FOR PCT MEASUREMENTS}

The last section indicates the importance of selecting an optimized measuring instrument to determine the proton energy with sufficient accuracy that small density changes can be detected. Tracking of individual protons requires the following proton measurements:

- location, before and after the object, to few hundred $\mu \mathrm{m}$;

- entrance and exit angles to much better than the MCS angle $\theta_{\mathrm{MCS}} \approx 1^{\mathrm{o}}$;

- average energy $\langle\mathrm{E}\rangle$ to better than $1 \%$.

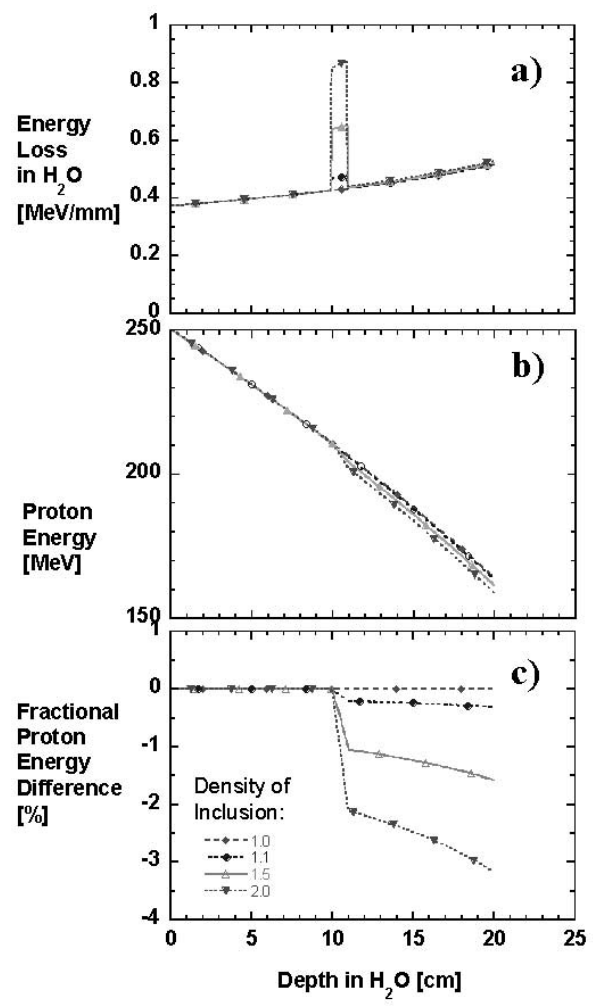

Fig. 4. Proton energy loss in water as a function of penetration depth for 250 $\mathrm{MeV}$ protons, with a $1 \mathrm{~cm}$ long inclusion of varying density at a depth of 10 $\mathrm{cm}$. The densities chosen are $\rho=1,1.1,1.5,2 \mathrm{~g} / \mathrm{cm}^{2}$. (a) Energy loss in 1 mm. (b) Proton energy. (c) Fractional energy difference relative to water ( $\rho=$ 1.0 ). Although the difference in energy loss is substantial locally, the effect of changed density is barely visible at a depth of $20 \mathrm{~cm}$ (see Table I). A density of $\rho=1.5$ corresponds to bone, and different soft tissues have $\rho \sim 0.9-1.1$.

TABLE I

VAlue of Proton Parameters After $20 \mathrm{~cm}$ of Water With a $1 \mathrm{~cm}$ INCLUSION OF DENSITY $\rho$ AT A DEPTH OF $10 \mathrm{~cm}$

\begin{tabular}{|c|c|c|c|}
\hline $\begin{array}{c}\mathbf{\rho}^{\mathbf{I}} \\
{\left[\mathbf{g} / \mathbf{c m}^{\mathbf{2}}\right]}\end{array}$ & $\begin{array}{c}\text { Energy } \\
{[\mathbf{M e V}]}\end{array}$ & $\begin{array}{c}\text { Range } \\
{[\mathbf{c m}]}\end{array}$ & $\begin{array}{c}\text { TOF } \\
{[\mathbf{p ~ s ]}}\end{array}$ \\
\hline 1.0 & 164.1 & 38.2 & 1309 \\
\hline 1.1 & 163.6 & 38.1 & 1311 \\
\hline 1.5 & 161.5 & 37.7 & 1317 \\
\hline 2.0 & 158.9 & 37.2 & 1325 \\
\hline
\end{tabular}

Silicon strip detectors (SSD) can provide position resolution of $50 \mu \mathrm{m}$ or better, and using a stack of two detectors or an SSD telescope will satisfy the requirement for angle $\theta_{\mathrm{MCS}} \approx 1^{\circ}$. The most exacting requirement is the good energy (or range) measurement. The loss of energy in the proton beam is equivalent to the attenuation of the photons in XCT, and is the primary parameter in the measurement. Here the determination of the average energy can be improved with statistics, because the error on the average energy $\sigma_{\langle\mathrm{E}\rangle}$ decreases with an increase the number of protons measured

$$
\sigma_{\langle\mathrm{E}\rangle}=\frac{\sigma_{E}}{\sqrt{N}}
$$

where $\sigma_{\mathrm{E}}$ is the energy uncertainty of a single measurement. A practical limit to improving the energy determination is the dose to the patient. The dose $\mathrm{D}$ is the absorbed energy per mass, and 
thus only a function of the fluence N/A, where $\mathrm{N}$ is the number of protons and $\mathrm{A}$ the area

$$
\mathrm{D}=\frac{\mathrm{N}}{\mathrm{A}} \cdot \frac{d E}{d x}
$$

Inside the imaged object, one can imagine a small volume ("voxel"), which one wants to resolve. It has a linear dimension $d$ with a density difference $\Delta \rho$ relative to the object. To achieve a significant energy measurement in this voxel, one needs $\mathrm{N}$ protons, which will deposit a dose Dv ... For a $1 \mathrm{~mm}^{3}$ voxel of size, if one employed $10^{6} 200 \mathrm{MeV}$ protons the dose would be about $70 \mathrm{mGy}$ (i.e., about equal to the standard average target dose for XCT). If this is adequate in terms of precision, the dose is acceptable, particularly for radiation therapy patients, who are about to receive much larger doses in the course of their treatment.

\section{PReliminary PRT Data}

A recent PRT study [6], [7] was made using state-of-the-art silicon strip detectors (SSDs), which measure the energy and position of individual protons. The SSDs provide information about the position of the particle track from the strip-hit information, and about the particle's energy via the energy deposition measured in each detector. This system, described in greater detail in [22], [24], permits measurements of the lateral proton position to about $50 \mathrm{~m}$ and determination of the energy of protons in the $20-300 \mathrm{MeV}$ range. The proton energy is derived from the specific energy deposition in each SSD using the time over threshold (TOT) signal as described in [24], [25]. This is possible due to the relative steep energy dependence of the stopping power (Fig. 1(b)). Using the expected and experimentally confirmed TOT versus energy curve, the energy resolution $\sigma_{\mathrm{E}} / \mathrm{E}$ below $40 \mathrm{MeV}$ is found to be on the order of $15 \%$ and increases to about $25 \%$ at $250 \mathrm{MeV}$. While this is not good enough for the final pCT system, it can be used to gain valuable experience in data reconstruction and simulation. The setup for the initial experiment, described in detail in [7], was installed on the research beam line of the medical proton synchrotron at Loma Linda University Medical Center [1]. A monochromatic $250 \mathrm{MeV}$ proton beam was degraded by a $25.4 \mathrm{~cm}$ thick wax block to a mean energy of about $130 \mathrm{MeV}$. After a drift distance of $25 \mathrm{~cm}$, the beam encountered the image object, a $5.0 \mathrm{~cm}$ long hollow aluminum cylinder, of outer diameter $\mathrm{OD}=3.0 \mathrm{~cm}$, and inner diameter ID $=0.68 \mathrm{~cm}$. Behind the object, protons were individually detected by two silicon detector modules, each consisting of a pair of single-sided SSDs with strips oriented at right angle to each other. These detectors, located immediately behind and $27 \mathrm{~cm}$ downstream from the object, served to measure the spatial coordinates, $\mathrm{x}$ and $\mathrm{y}$, and energy of the protons that either passed by or traversed the object. In addition, they allow reconstruction of the exit angle of the protons. Proton transmission images were calculated for each SSD module by averaging the proton energy over $\sim 10^{6}$ individual events. The three-dimensional plot in Fig. 5 shows the spatial distribution of average energy in the upstream module with single strip resolution (pixel size approximately $0.2 \times 0.2 \mathrm{~mm}^{2}$ ). The image of

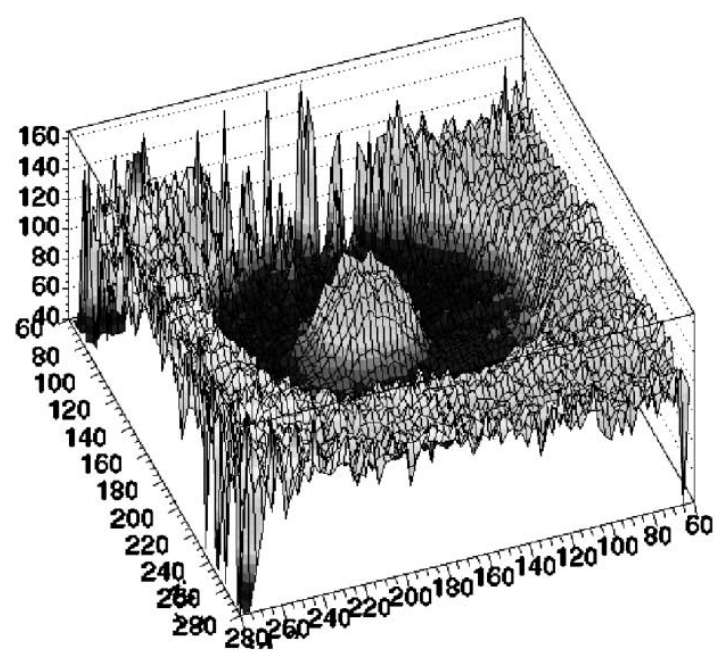

Fig. 5. Spatial distribution of the average energy of protons with single-strip resolution at the level of the upstream SSD module [7]. The increased energy loss in the pipe is clearly visible.

the phantom projection is clearly seen in the spatial energy distribution. Note that the depth of the structure in Fig. 5 is directly proportional to the energy loss in the aluminum object and thus is proportional to the product of its length and density. Fig. 5 thus demonstrates the possibility of image formation based on the spatial measurement of proton energy loss in an object.

\section{GEANT4 SimUlations}

To better understand the features of the proton transmission images presented in Section IX, simulations with the Monte Carlo code GEANT4 were performed [26]. The GEANT4 code has proved its ability to faithfully simulate the interaction of protons down to low energy [27]. Here, the code was used to define cuts on the data to optimize spatial resolution and contrast of the proton images. Details of the simulations are given in [28], which showed excellent agreement between data and simulated angular distributions. The angular spread for protons, which traverse the pipe in its entirety, is about $5^{\circ}$, and for protons, which miss the pipe completely about $1^{\circ}$. The difference between the distributions is caused by the increased multiple Coulomb scatters in the pipe [see (3)], an indication that the simulations can be used further to explore the usefulness of angular cuts. With the selected cut at 0.025 (about $1.5^{\circ}$ ), protons with larger angular divergence are rejected, which eliminates about $50 \%$ of the protons passing through the object. The result of this cut on the simulated energy profile in the $x$ and $y$ silicon planes close to the object is shown in Fig. 6. The location of the profile corresponds to a slice across the center of the pipe in Fig. 5. The simulated energy profile [Fig. 6(a)] agrees well with the measured outline of the object (and with the measured energy profile, not shown here, within the limits of the calibration). The mean simulated energy of protons transmitted through the hole is about $10 \%$ lower than that for protons passing outside the object. Furthermore, in agreement with the measured energy profile, both the inner and outer walls of the pipe appear blurred in the energy profile. These image features can be explained by "migration" 


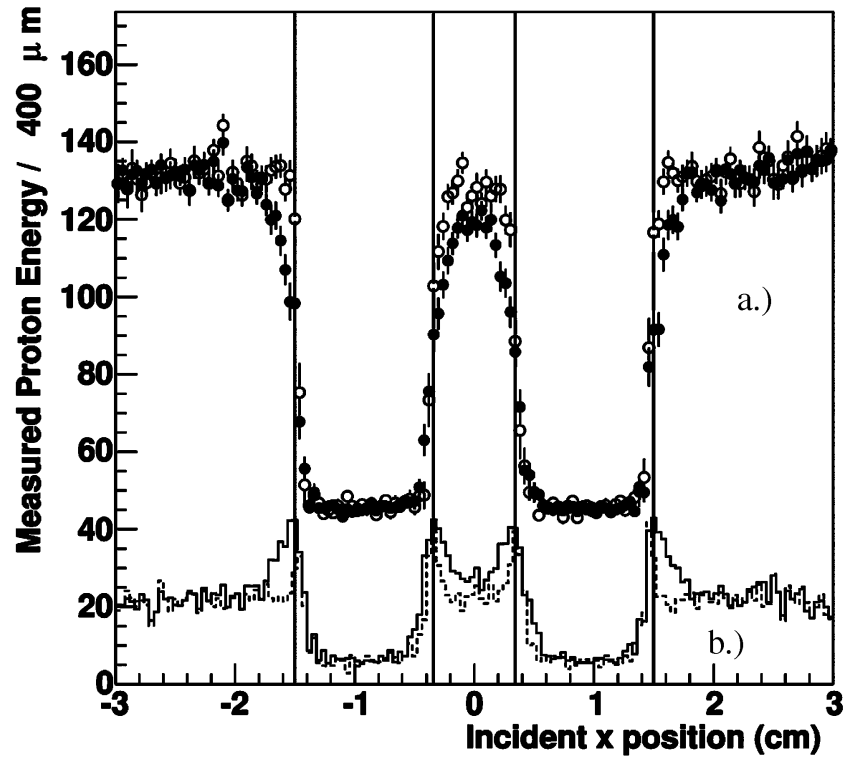

Fig. 6. (a) Monte Carlo simulation of the average energy profile of the first SSD module in a $400 \mu \mathrm{m}$ wide slice through the center of the object in Fig. 5. The dashed vertical lines indicate the relative position of the object with respect to the SSD plane. (b) The rms deviation of the proton energy. Note the increase in the rms at the interfaces between object and air. The closed symbols and the solid histogram are for all particles, and the open circles and the dashed histogram are for protons within the angular cut of 0.025 . The improvement in image sharpness is seen in the more vertical interfaces, filling in the hole, and the reduction of the width of the region with increased energy rms at the pipe-air interface [6].

of protons from the object into the surrounding space due to multiple Coulomb scattering. This assumption is supported by the distribution of the energy spread (rms), shown in Fig. 6(b), which is larger at the interfaces between the object and the surrounding air, indicating a mixture of protons with and without energy loss in this region. Protons with lower energies entered the object trough the front surface and exited through the sides. These protons "scattering out" also cause the blurred edge profile. The angular cut removes most of the migrating protons and sharpens the image considerably. In Fig. 6(a), the open symbols correspond to the energy profile with angular cut applied. The edges become sharp, with a transition from $\mathrm{Al}$ to air almost within a bin of $400 \mu \mathrm{m}$. In addition the central hole fills in. The energy rms plot Fig. 6(b) (in red: without angular cut, in green with angular cut) indicates that the rms is increased just in one bin, otherwise it is constant across the region of Al pipe and air, respectively. Thus there is strong evidence that the resolution of the pCT image can be improved by cuts on the exit angle.

\section{CONCLUSION}

The renewed interest in imaging with pCT is based on several factors: operation of new accelerators for proton therapy, need to support cancer treatment with protons, academic interest, new simulation codes and the use of new detector systems. Exploratory PRT experiments give valuable insight into the requirements for imaging with protons. The superior position resolution and ease of operation of silicon strip detectors will be an advantage, and should be backed up with an energy measurement with about $1 \%$ resolution to minimize the dose to the patient in $\mathrm{pCT}$. The simulations with the GEANT4 program describe the features of the images well, e.g., the influence of multiple scattering and proton migration on the energy and position resolution. It is possible to mediate the blurring effects of multiple scattering by measuring the exit angles of individual protons with the silicon telescope and applying appropriate cuts.

The concepts and data presented here only indicate the potential of pCT. Unlike XCT, the measurement is of the energy and angular deflection of individual protons. This is a whole new set of parameters and concepts for medical imaging scientists to explore. The power of proton radiotherapy is the potential to irradiate very specific points in the body with minimal dose to healthy tissue. This potential will not be realized if the data used in treatment plan is compromised by approximations used in the treatment planning. It is important that the relationship between the XCT data and the pCT data be known precisely. Given the wide availability of XCT in the clinical world, XCT will most likely be the starting point of almost all treatment plans, until pCT has been proven to be practical.

\section{ACKNOWLEDGMENT}

The authors would like to thank the organizers of the 2002 NSS/MIC Symposium for a very stimulating meeting.

\section{REFERENCES}

[1] J. M. Slater, J. O. Archambeau, D. W. Miller, M. I. Notarus, W. Preston, and J. D. Slater, "The proton treatment center at Loma Linda university medical center: Rationale for and description of its development," Int. J. Radiat. Oncol. Biol. Phys., vol. 22, pp. 383-389, 1992.

[2] U. Schneider and E. Pedroni, "Proton radiography as a tool for quality control in proton therapy," Med. Phys., vol. 22, pp. 353-363, 1995.

[3] Nat. Inst. Standards Technol., PSTAR Database [Online]. Available: http://physics.nist.gov/PhysRefData/Star/Text/PSTAR.html

[4] H. Bichsel, Passage of Charged Particles Through Matter. New York: McGraw-Hill, 1972, pp. 8-142-8-189.

[5] D. E. Groom, Ed., "Review of particle physics," in Eur. Phys. J., 2000, vol. C15, pp. 1-878.

[6] H. F.-W. Sadrozinski et al., "Issues in proton CT," Nucl. Instrum. Methods A, vol. 511, pp. 275-281, 2003.

[7] L. R. Johnson et al., "Initial studies from on proton CT using a silicon strip detector telescope," in Proc. RESMDD'02, SCIPP 02/32, Florence, Italy, July 2002.

[8] S. Peggs, "Proton therapy accelerators," presented at the Proc. 2002 IEEE NSS/MIC, Norfolk, VA, Nov. 2002, invited talk M2-2.

[9] U. Schneidert, E. Pedroni, and A. Lomax, "The calibration of CT hounsfield units for radiotherapy treatment planning," Phys. Med. BioI., vol. 41, pp. 111-124, 1996.

[10] R. R. Wilson, "Radiological use of fast protons," Radiol., vol. 47, p. 487, 1946.

[11] A. M. Cormack. Nobel Lecture. [Online]. Available: http://www.nobel.se/medicine/laureates/1979/cormack-lecture.pdf

[12] M. Goitein, "Three-dimensional density reconstruction from a series of two-dimensional projections," Nucl. Instrum. Methods, vol. 101, pp. $509-518,1972$.

[13] A. M. Cormack and A. M. Koehler, "Quantitative proton tomography," Phys. Med. Biol., vol. 21, pp. 560-569, 1976.

[14] K. M. Hanson et al., "Proton computed tomography with human specimens," Phys. Med. Biol., vol. 27, pp. 25-36, 1982.

[15] U. Schneider and A. Tourovsky, "Range-uncertainty imaging for obtaining dose perturbations in proton therapy," IEEE Trans. Nucl. Sci., vol. 45 , p. 2309 , Oct. 1998.

[16] T. J. Satogata, T. Satogata, T. Bacarian, S. Peggs, A. G. Ruggiero, and F. A. Dilmanian, "Reduced dose of proton CT compared to X-Ray CT in tissue-density variation sensitivity," presented at the Proc. 2002 IEEE NSS/MIC, Norfolk, VA, Nov. 2002, Poster M10-204.

[17] U. Schneider, "Proton Radiography: A Tool for Quality Control in Proton Therapy," Ph.D. dissertation, ETH, Zurich, Switzerland, 1994. 
[18] P. Pemler, "Entwicklung, Bau und Test eines Detektorsystems für in vivo protonenradiographie FROG," Ph.D. dissertation, LMU, Munich, Germany, 1997.

[19] P. Zygmanski, "Proton Cone-Beam Computed Tomography," Ph.D. dissertation, Univ. Massachusetts Phys. Dept., Amherst, MA, 1998.

[20] U. Schneider and E. Pedroni, "Multiple Coulomb scattering and spatial resolution in proton radiography," Med. Phys., vol. 21, no. 11, pp. 1657-1663, Nov. 1994.

[21] D. C. Williams, "The Most Likely Path of an Energetic Charged Particle Through a Uniform Medium,", SCIPP 03/07.

[22] B. Keeney et al., "A silicon telescope for applications in nanodosimetry," IEEE Trans. Nucl. Sci., vol. 49, pp. 1724-1727, Aug. 2002.

[23] B. Keeney, "A Silicon Telescope for Applications in Nanodosimetry," Univ. California-Santa Cruz, Physics Senior Thesis 2002, Santa Cruz, CA. SCIPP 02/16.
[24] E. do Couto e Silva et al., "Results from the beam test of the engineering model of the GLAST LAT," Nucl. Instrum. Methods A., vol. 474/1, pp. 19-37, 2001.

[25] R. P. Johnson, P. Poplevin, H. F.-W. Sadrozinski, and E. Spencer, "An amplifier-discriminator chip for the GLAST silicon-strip tracker," IEEE Trans. Nucl. Sci., vol. 45, pp. 927-932, June 1998.

[26] S. Agostinelli et al., "GEANT4-A simulation toolkit," Nucl. Instrum. Methods A, vol. 506, pp. 250-303, 2003.

[27] GEANT4 Workshop at SLAC (2002, Feb. 18-22). [Online]. Available: http://geant4.slac.stanford.edu/UsersWorkshop/

[28] L. R. Johnson et al., "Monte carlo studies of proton CT using a silicon strip detector telescope," presented at the Proc. 2002 IEEE NSS/MIC, Norfolk, VA, Nov. 2002, Poster M6-2, SCIPP 02/35. 